\title{
Topological Characterization of the Symmetrical Structure of Bismuth Tri-Iodide
}

\author{
Muhammad Imran 1,2,*, Muhammad Arfan Ali ${ }^{3}$, Sarfraz Ahmad ${ }^{3}$, \\ Muhammad Kamran Siddiqui ${ }^{1,4}$ and Abdul Qudair Baig ${ }^{5}$ \\ 1 Department of Mathematical Sciences, United Arab Emirates University, Al Ain, P. O. Box 15551, \\ United Arab Emirates \\ 2 Department of Mathematics, School of Natural Sciences (SNS), National University of Sciences and \\ Technology (NUST), Sector H-12, Islamabad 44000, Pakistan \\ 3 Department of Mathematics, Comsats Institute of Information Technology, Lahore 54000, Pakistan; \\ arfanali709@gmail.com (M.A.A.); sarfrazahmad@ciitlahore.edu.pk (S.A.) \\ 4 Department of Mathematics, Comsats Institute of Information Technology, Sahiwal 57000, Pakistan; \\ kamransiddiqui75@gmail.com \\ 5 Department of Mathematics, The University of Lahore, Pakpattan Campus 57400, Pakistan; \\ aqbaig1@gmail.com \\ * Correspondence: imrandhab@gmail.com
}

Received: 14 May 2018; Accepted: 1 June 2018; Published: 4 June 2018

\begin{abstract}
The bismuth tri-iodide $\left(\mathrm{BiI}_{3}\right)$ is an inorganic compound. It is the result of the response of bismuth and iodine, which has inspired enthusiasm for subjective inorganic investigation. The topological indices are the numerical invariants of the molecular graph that portray its topology and are normally graph invariants. In 1975, Randic presented, in a bond-added substance, a topological index as a descriptor for portraying subatomic branching. In this paper, we investigate the precious stone structure of bismuth tri-iodide chain and sheet. Moreover, exact formulas of degree-based added-substance topological indices principally the first, second, and hyper Zagreb indices, the general Randic index, the geometric-arithmetic index, the fourth atom-bond connectivity index, and the fifth geometric arithmetic index of the subatomic graph of bismuth tri-iodide for both chain and sheet structures are determined.
\end{abstract}

Keywords: molecular descriptors; crystal structures; bismuth tri-iodide; atom-bond connectivity index; geometric-arithmetic index; zagreb type indices

\section{Introduction}

Graph theory is a multidimensional subject in light of its huge applications in both unadulterated and connected science. It is viable in terms of displaying and planning crystal structures, complex systems, and synthetic graphs. There are number of chemical compounds that are organic and inorganic and that have applications in commercial, industrial, and laboratory contexts and in daily life. A relationship exists between synthetic mixes and their atomic structures. Graph theory is an effective territory of arithmetic that has a tremendous scope of utilizations in numerous areas of science, such as chemistry, software engineering, electrical, and electronics. Chemical graph theory is a branch of science in which proficient apparatuses of graph theory are utilized to graphically demonstrate concoctions.

The physical structure of strong materials depends generally on the courses of action of atoms, particles, or atoms that make up the strong holding powers between them. The crystal structure, also referred to as crystalline material or crystalline strong is made out of unit cells, and molecules are organized in $3 \mathrm{D}$ on a grid. The schemes of atoms or crystalline material are vital in determining the 
conduct and properties of a material, such as metals, composites, and artistic materials. The unit cell is the smallest auxiliary unit that can clarify the precious stone structure. The redundancy of the unit cell creates the entire precious stone.

Let $G=(V, E)$ be a subatomic diagram, where $V$ is the arrangement of the vertex, called atoms, and $E$ is the edge set, called the chemical bond. The degree $d_{v}$ of $v$ is the quantity of edges of $G$ occurrence with $v$. An atomic diagram can be informed by a polynomial, a numerical esteem, an eigenvalue, a topological index, and so forth. There are certain types of topological indices mostly distance-based, degree-based, and distance-based indices. This article is completely dedicated to managing degree-based additive topological indices. A topological index is a number that depicts vital and valuable information about molecular structure. It is a numerical invariant of a subatomic graph and is very valuable we're comparing their bioactivity and physio-synthetic properties.

In 1975, the very first degree-based index was introduced by Randić [1]:

$$
R_{-\frac{1}{2}}(G)=\sum_{e f \in E(G)} \frac{1}{\sqrt{d_{e} \times d_{f}}} .
$$

In 1988, Bollobás et al. [2] and Amic et al. [3] independently proposed the general Randic index. For more details about the important results and chemical properties of the Randić index, we recommend [4]. The general Randic index is defined as

$$
R_{\alpha}(G)=\sum_{e f \in E(G)}\left(d_{e} \times d_{f}\right)^{\alpha}, \quad \alpha=1,-1, \frac{1}{2}, \frac{-1}{2} .
$$

Estrada et al. [5] introduced the atom-bond connectivity index:

$$
A B C(G)=\sum_{e f \in E(G)} \sqrt{\frac{d_{e}+d_{f}-2}{d_{e} \times d_{f}}}
$$

In 1972, both the first and second Zagreb indices were formulated by Gutman [6] as follows:

$$
\begin{aligned}
& M_{1}(G)=\sum_{e f \in E(G)}\left(d_{e}+d_{f}\right) \\
& M_{2}(G)=\sum_{e f \in E(G)}\left(d_{e} \times d_{f}\right) .
\end{aligned}
$$

For more details about Zagreb indices, their properties, and important results, see [7-9]. Shirdel et al. introduced the hyper Zagreb index in 2013 [10]. The mathematical representation of this index is as follows:

$$
H M(G)=\sum_{e f \in E(G)}\left(d_{e}+d_{f}\right)^{2}
$$

The fourth version of the atom-bond connectivity index $A B C_{4}$ of a graph $G$ was introduced by Ghorbhani et al. [11] and is defined as follows:

$$
A B C_{4}(G)=\sum_{e f \in E(G)} \sqrt{\frac{S_{e}+S_{f}-2}{S_{e} \times S_{f}}}
$$

where $S_{e}=\sum_{e f \in E(G)} d_{f}$ and $S_{f}=\sum_{e f \in E(G)} d_{e}$. 
Vukicevic et al. [12] defined the geometric arithmetic index $G A$ of a graph $G$ as follows:

$$
G A(G)=\sum_{e f \in E(G)} \frac{2 \sqrt{d_{e} \times d_{f}}}{d_{e}+d_{f}} .
$$

Another well known molecular descriptor is the fifth version of the geometric arithmetic index $G A_{5}$ of a graph $G$. It was introduced by Graovac et al. [13] and is defined as follows:

$$
G A_{5}(G)=\sum_{e f \in E(G)} \frac{2 \sqrt{S_{e} \times S_{f}}}{S_{e}+S_{f}} .
$$

\section{Applications of Topological Indices}

The Randic index is a topological descriptor related to a great deal of synthetic qualities of atoms and was discovered parallel to processing the boiling point and Kovats constants of the particles. The particle bond network $(A B C)$ index connects to the security of direct alkanes and stretched alkanes and is used to process the strain vitality of cyclo alkanes [14,15]. In terms of physico-concoction properties, the $G A$ index has prescient control superior to the prescient energy of the Randic connectivity index [16]. The first and second Zagreb indices were found to be helpful for calculation of the aggregate $\pi$-electron energy of the particles inside particular rough articulations [17]. These are among the graph invariants proposed for the estimation of skeletons of stretching of carbon atoms [18].

During the last two decades, analysts created substance diagrams, and they arranged and processed particular indices. W. Gao and M. R. Farahani calculated degree-based indices of synthetic structures by utilizing an edge-separated technique [19]. Gao et al. [20,21] created concoction structures in medications and processed overlooked topological indices. As of late, Baig et al. [22] computed and graphically portrayed topological descriptors of concoction graphs of carbon graphite and precious stone cubic carbon structures. Different utilizations of atomic descriptors of subatomic diagrams and systems are given in the reference list and the references in [23,24]. These applications and literature reviews inspired us to investigate new substance diagrams and gem structures and process their topological records.

\section{Methods}

For the computation of our results, we utilized a strategy for combinatorial registering, a vertex partition strategy, an edge partition technique, graph hypothetical instruments, scientific systems, a degree-counting strategy, and a degrees of neighbors strategy. In addition, we used Matlab for scientific estimations and confirmations. We likewise utilized Maple for plotting numerical results.

\section{Bismuth Tri-Iodide}

The bismuth tri-iodide $\mathrm{BiI}_{3}$ is an outstanding inorganic compound. This dark gray solid is the product of a reaction of bismuth and iodine, which was once of interest in qualitative inorganic analysis [25]. It has been demonstrated over the years that Bi-doped glass optical strands are among the most promising dynamic laser media. Different kinds of Bi-doped optical strands have been created as a result and have been used to build Bi-doped fiber lasers and optical speakers [26]. Layered $\mathrm{BiI}_{3}$ precious stone is thought to be a three-layered stacking structure, where bismuth atom planes are sandwiched between iodide particle planes, which shape the succession $I-B i-I$ planes [27]. The periodic stacking of three layers forms rhombohedral $\mathrm{Bi}_{3}$ crystal with $R-3$ symmetry $[28,29]$. The progressive stacking of one $I-B i-I$ layer shapes the hexagonal structure with symmetry [30]. A solitary precious stone of $\mathrm{BiI}_{3}$ has been integrated by Nason and Keller [31]. The graph of a solitary unit of bismuth tri-iodide 
contains six 4-cycles, of which two are on the main, two are in the center, and two are at the base. The bismuth tri-iodide can be organized either straightly (chain) or in a sheet frame [32].

\subsection{Results for Bismuth Tri-Iodide Chain $\left(m-\mathrm{BiI}_{3}\right)$}

The linear arrangement of bismuth tri-iodide with $m$ unit cells is called $m$-bismuth tri-iodide chain. The unit cell of bismuth tri-iodide $\mathrm{BiI}_{3}$ is illustrated in Figure 1 . The cardinality of vertices and edges of bismuth tri-iodide chain $m-\mathrm{BiI}_{3}$ are $6(3 m+2)$ and $12(2 m+1)$, respectively. In $m-\mathrm{BiI}_{3}$ chain, the 1 degree vertices are $4(m+2)$, the 2 degree vertices are $2(5 m+1)$, and the 6 degree vertices are $2(2 m+1)$. The edge set of $m-\mathrm{BiI}_{3}$ chain is divided into two partitions based on the degree of end vertices. The first edge partition contains $4 m+8$ edges $u v$, where $d_{e}=1$ and $d_{f}=6$. The second edge partition contains $20 m+4$ edges $u v$, where $d_{e}=2$ and $d_{f}=6$. Table 1 shows the edge partition of bismuth tri-iodide $m-\mathrm{Bi}_{3}$ for $m>1$.

The chain bismuth tri-iodide for $m=3$ is formed by combining one unit cell of $\mathrm{BiI}_{3}$ three times together as shown in Figure 2.

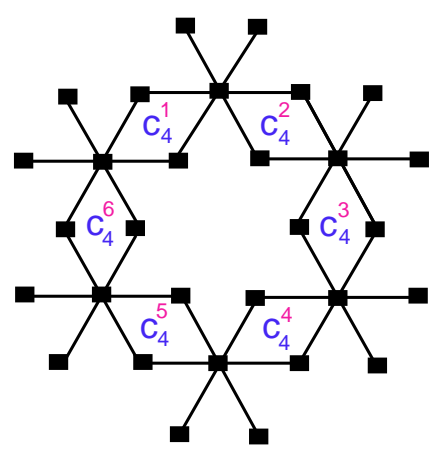

$$
\begin{aligned}
& \text { Main Cycles: } \mathrm{C}_{4}^{1}, \mathrm{C}_{4}^{2} \\
& \text { Central Cycles: } \mathrm{C}_{4}^{3}, \mathrm{C}_{4}^{6} \\
& \text { Base Cycles: } \mathrm{C}_{4}^{4}, \mathrm{C}_{4}^{5}
\end{aligned}
$$

Figure 1. The unit cell of bismuth tri-iodide.

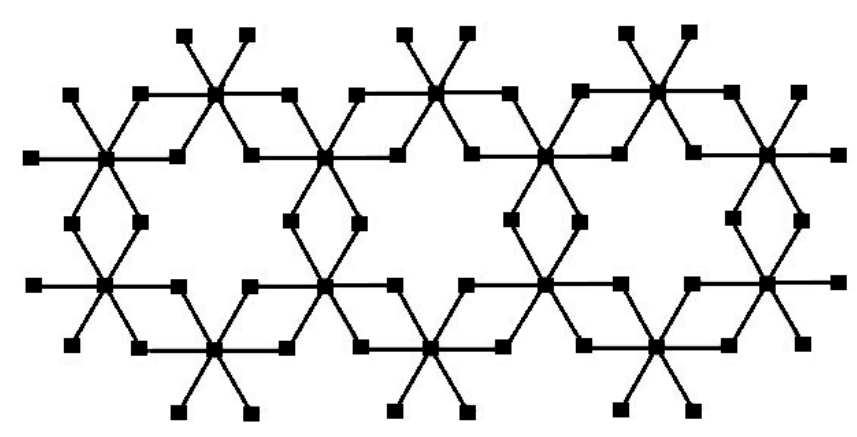

Figure 2. The chain bismuth tri-iodide for $m=3$.

Table 1. Edge partition of $m-\mathrm{BiI}_{3}$.

\begin{tabular}{ccc}
\hline$\left(\boldsymbol{d}_{\boldsymbol{e}}, \boldsymbol{d}_{f}\right)$ & $(\mathbf{1}, \mathbf{6})$ & $(\mathbf{2}, \mathbf{6})$ \\
\hline Number of Edges & $4 m+8$ & $20 m+4$ \\
\hline
\end{tabular}

In the next step, we computed the atom-bond connectivity index, the general Randić $R_{\alpha}$ index for $\alpha=\left\{-1,1,-\frac{1}{2}, \frac{1}{2}\right\}$, the geometric arithmetic index, the fourth atom-bond connectivity index $A B C_{4}$, the fifth geometric arithmetic index $G A_{5}$, and the first, second, and hyper Zagreb indices. We present these results graphically for the crystal structure of bismuth tri-iodide for both chain and sheet.

\section{- Atom-bond connectivity index.}


Let $G$ represent the molecular graph of $m-B i I_{3}$. Then, from Table 1, the atom-bond connectivity index is computed as

$$
\begin{aligned}
A B C(G) & =\sum_{e f \in E(G)} \sqrt{\frac{d_{e}+d_{f}-2}{d_{e} \times d_{f}}} \\
A B C(G) & =4(m+2) \sqrt{\frac{1+6-2}{1 \times 6}}+4(5 m+1) \sqrt{\frac{2+6-2}{2 \times 6}} \\
& =\frac{2 \sqrt{2}}{3}((\sqrt{15}+15) m+2 \sqrt{15}+3) .
\end{aligned}
$$

- The geometric arithmetic index $G A\left(m-B i I_{3}\right)$.

Let $G$ be the graph of $m-B i I_{3}$. Now, using Table 1, the geometric arithmetic index is computed as

$$
\begin{aligned}
G A(G) & =\sum_{e f \in E(G)} \frac{2 \sqrt{d_{e} d_{f}}}{d_{e}+d_{f}} \\
G A(G) & =4(m+2) \frac{2 \sqrt{1 \times 6}}{1+6}+4(5 m+1) \frac{2 \sqrt{2 \times 6}}{2+6} \\
& =\frac{2 \sqrt{3}}{7}((4 \sqrt{2}+35) m+8 \sqrt{2}+7) .
\end{aligned}
$$

- The general Randić index $R_{\alpha}\left(m-B i I_{3}\right)$.

Let $G$ be the graph of $m-B i I_{3}$. Now, using Table 1 , the general Randić index for $\alpha=1$ is

$$
\begin{aligned}
& R_{1}(G)=\sum_{e f \in E(G)}\left(d_{e} \times d_{f}\right) \\
& R_{1}(G)=4(m+2)(1 \times 6)+4(5 m+1)(2 \times 6)=264 m+24 .
\end{aligned}
$$

For $\alpha=-1$,

$$
\begin{aligned}
& R_{-1}(G)=\sum_{e f \in E(G)} \frac{1}{\left(d_{e} \times d_{f}\right)} \\
& R_{-1}(G)=4(m+2) \frac{1}{(1 \times 6)}+4(5 m+1) \frac{1}{(2 \times 6)}=\frac{7 m+5}{3} .
\end{aligned}
$$

For $\alpha=\frac{1}{2}$

$$
\begin{aligned}
R_{\frac{1}{2}}(G) & =\sum_{e f \in E(G)} \sqrt{\left(d_{e} \times d_{f}\right)} \\
R_{\frac{1}{2}}(G) & =4(m+2) \sqrt{(1 \times 6)}+4(5 m+1) \sqrt{(2 \times 6)} \\
& =4((10 \sqrt{3}+\sqrt{6}) m+2(\sqrt{3}+\sqrt{6})) .
\end{aligned}
$$


For $\alpha=-\frac{1}{2}$

$$
\begin{aligned}
R_{-\frac{1}{2}}(G) & =\sum_{e f \in E(G)} \frac{1}{\sqrt{\left(d_{e} \times d_{f}\right)}} \\
R_{-\frac{1}{2}}(G) & =4(m+2) \frac{1}{\sqrt{(1 \times 6)}}+4(5 m+1) \frac{1}{\sqrt{(2 \times 6)}} \\
& =\frac{2}{3}((5 \sqrt{3}+\sqrt{6}) m+\sqrt{3}+2 \sqrt{6}) .
\end{aligned}
$$

- $\quad$ First, second, and hyper Zagreb indices.

Let $G$ be the graph of $m-B i I_{3}$. Now, using Table 1, the first, second, and hyper Zagreb indices are computed as

$$
\begin{aligned}
& M_{1}(G)=\sum_{e f \in E(G)}\left(d_{e}+d_{f}\right) \\
& M_{1}(G)=4(m+2)(1+6)+4(5 m+1)(2+6)=188 m+88 \\
& M_{2}(G)=\sum_{e f \in E(G)}\left(d_{e} \times d_{f}\right) \\
& M_{2}(G)=4(m+2)(1 \times 6)+4(5 m+1)(2 \times 6)=264 m+96 .
\end{aligned}
$$

The hyper Zagreb index is computed as

$$
\begin{aligned}
& H M(G)=\sum_{e f \in E(G)}\left(d_{e}+d_{f}\right)^{2} \\
& H M(G)=4(m+2)(1+6)^{2}+4(5 m+1)(2+6)^{2}=1474 m+684 .
\end{aligned}
$$

Table 2 demonstrates the edge distribution in light of the degree sum of the end vertices of each edge. We find an exact formula for the fourth atom-bond connectivity index and the fifth geometric arithmetic index using Table 2.

Table 2. Edge partition of $m-\mathrm{BiI}_{3}$ based on the degree sum of end vertices of each edge.

\begin{tabular}{cccc}
\hline$\left(S_{e}, S_{f}\right)$ & $(\mathbf{6}, \mathbf{1 0})$ & $(\mathbf{1 0}, \mathbf{1 2})$ & $(\mathbf{1 2}, \mathbf{1 2})$ \\
\hline Number of Edges & $4 m+8$ & $8 m+16$ & $12 m-12$ \\
\hline
\end{tabular}

- The fourth atom-bond connectivity index $A B C_{4}\left(m-B i I_{3}\right)$.

Let $G$ be the graph of $m-B_{3}$. Now, using Table 2, the fourth atom-bond connectivity index is computed as

$$
\begin{aligned}
A B C_{4}(G) & =\sum_{e f \in E(G)} \sqrt{\frac{S_{e}+S_{f}-2}{S_{e} \times S_{f}}} \\
A B C_{4}(G) & =4(m+2) \sqrt{\frac{6+10-2}{6 \times 10}}+8(m+2) \sqrt{\frac{10+12-2}{10 \times 12}}+12(m-1) \sqrt{\frac{12+12-2}{12 \times 12}} \\
& =\frac{\sqrt{2}}{15}((20 \sqrt{3}+15 \sqrt{11}+2 \sqrt{105}) m+40 \sqrt{3}-15 \sqrt{11}+4 \sqrt{105}) .
\end{aligned}
$$


- The fifth geometric arithmetic index $G A_{5}\left(m-B i I_{3}\right)$.

Let $G$ be the graph of $m-B_{i}$. Now, using Table 2, the fifth geometric arithmetic index is computed as follows:

$$
\begin{aligned}
G A_{5}(G) & =\sum_{e f \in E(G)} \frac{2 \sqrt{S_{e} \times S_{f}}}{S_{e}+S_{f}} \\
G A_{5}(G) & =4(m+2) \frac{2 \sqrt{6 \times 10}}{6+10}+8(m+2) \frac{2 \sqrt{10 \times 12}}{10+12}+12(m-1) \frac{2 \sqrt{12 \times 12}}{12+12} \\
& =\frac{1}{11}((11 \sqrt{15}+16 \sqrt{30}+132) m+22 \sqrt{15}+32 \sqrt{30}-132) .
\end{aligned}
$$

\subsection{Results for Bismuth Tri-Iodide Sheet $\mathrm{BiI}_{3}(m \times n)$}

In this section, the topological additive indices based on the degree and sum of degree of bismuth tri-iodide sheet are computed. The linear arrangement of bismuth tri-iodide with $m n$ unit cells is called $m \times n$ bismuth tri-iodide sheet, where $m$ unit cells are taken as rows and $n$ unit cells are taken as columns. The graph of bismuth tri-iodide sheet for $m=2$ and $n=3$ is represented in Figure 3.

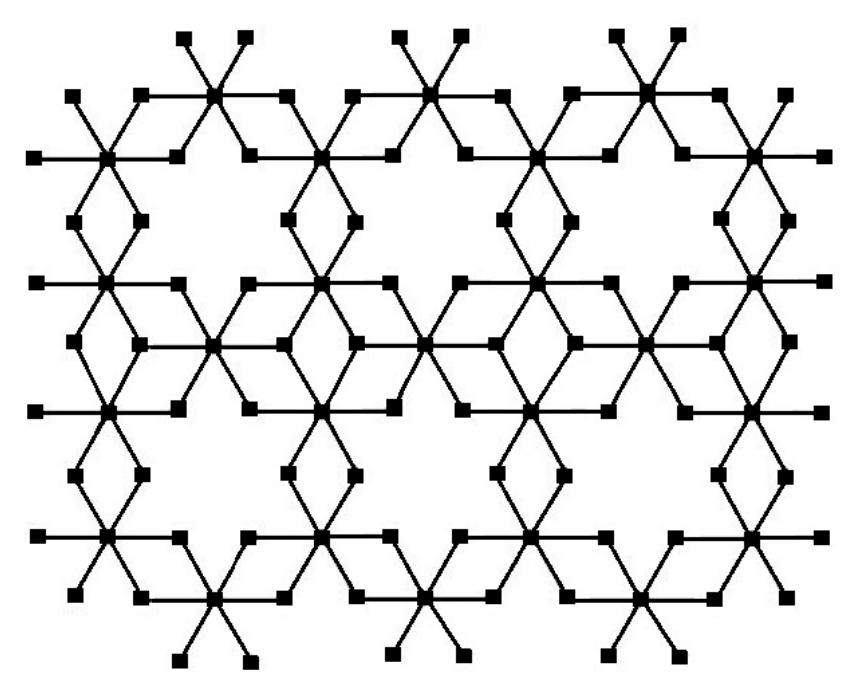

Figure 3. The sheet bismuth tri-iodide for $m=2$ and $n=3$.

The cardinality of vertices and edges of bismuth tri-iodide sheet $\mathrm{BiI}_{3}(m \times n)$ are $11 m n+10 m+$ $7 n+2$ and $18 m n+12 m+6 n$, separately. In $\mathrm{Bi}_{3}(m \times n)$ sheet, the 1 degree vertices are $4(m+n+1)$, the 2 degree vertices are $6 m n+4 m 4 n-2$, the 3 degree vertices are $2 m n-2 n$, and the 6 degree vertices are $3 m n+2 m+n$. The edge set of the $\mathrm{BiI}_{3}(m \times n)$ sheet is separated into three parcels in light of the level of the end vertices. The main edge parcel contains $4 m+4 n+4$ edges $u v$, where $d_{e}=1$ and $d_{f}=6$. The second edge segment contains $12 m n+8 m+8 n-4$ edges $u v$, where $d_{e}=2$ and $d_{f}=6$. The third edge segment contains $6 m n-6 n$ edges $u v$, where $d_{e}=3$ and $d_{f}=6$. Table 3 demonstrates the edge parcel of the bismuth tri-iodide sheet for $m, n>1$.

Table 3. Edge partition of $\mathrm{BiI}_{3}(m \times n)$ based on the degree of end vertices of each edge.

\begin{tabular}{cccc}
\hline$\left(d_{e}, d_{f}\right)$ & $(\mathbf{1}, \mathbf{6})$ & $(\mathbf{2}, \mathbf{6})$ & $(\mathbf{3}, \mathbf{6})$ \\
\hline Number of Edges & $4 m+4 n+4$ & $12 m n+8 m+8 n-4$ & $6 m n-6 n$ \\
\hline
\end{tabular}

- The atom-bond connectivity index $A B C\left(\operatorname{BiI}_{3}(m \times n)\right)$. 
Let $G$ be the graph of $\operatorname{BiI}_{3}(m \times n)$ with $m, n>1$. From the edge partition of $\operatorname{BiI}_{3}(m \times n)$, which is given in Table 3 , the atom-bond connectivity index is computed as

$$
\begin{aligned}
A B C(G) & =\sum_{e f \in E(G)} \sqrt{\frac{d_{e}+d_{f}-2}{d_{e} \times d_{f}}} \\
A B C(G) & =4(m+n+1) \sqrt{\frac{1+6-2}{1 \times 6}}+(12 m n+8 m+8 n-4) \sqrt{\frac{2+6-2}{2 \times 6}}+(6 m n-6 n) \sqrt{\frac{3+6-2}{3 \times 6}} \\
& =(6 \sqrt{2}+\sqrt{14}) m n+2(m+n)\left(2 \sqrt{2}+\frac{\sqrt{30}}{3}\right)-\sqrt{14} n-2 \sqrt{2}+\frac{2 \sqrt{30}}{3} .
\end{aligned}
$$

- The geometric arithmetic index $G A\left(B I_{3}(m \times n)\right)$.

Let $G$ be the graph of $\operatorname{BiI}_{3}(m \times n)$ with $m, n>1$. Now, using Table 3, the geometric arithmetic index is computed as follows:

$$
\begin{aligned}
G A(G) & =\sum_{e f \in E(G)} \frac{2 \sqrt{d_{e} \times d_{f}}}{d_{e}+d_{f}} \\
G A(G) & =4(m+n+1) \frac{2 \sqrt{1 \times 6}}{1+6}+(12 m n+8 m+8 n-4) \frac{2 \sqrt{2 \times 6}}{2+6}+(6 m n-6 n) \frac{2 \sqrt{3 \times 6}}{3+6} \\
& =2(2 \sqrt{2}+3 \sqrt{3}) m n+4(m+n)\left(\frac{7 \sqrt{3}+2 \sqrt{6}}{7}\right)-4 \sqrt{2} n-2 \sqrt{3}+\frac{8 \sqrt{6}}{7} .
\end{aligned}
$$

- The General Randić index $R_{\alpha}\left(\operatorname{BiI}_{3}(m \times n)\right)$.

Let $G$ be the graph of $\operatorname{BiI}_{3}(m \times n)$ with $m, n>1$. Now, using Table 3, the general Randić index is given:

For $\alpha=1$,

$$
\begin{aligned}
R_{1}(G) & =\sum_{e f \in E(G)}\left(d_{e} \times d_{f}\right) \\
R_{1}(G) & =4(m+n+1)(1 \times 6)+(12 m n+8 m+8 n-4)(2 \times 6)+(6 m n-6 n)(3 \times 6) \\
& =252 m n+120 m+12 n-24 .
\end{aligned}
$$

For $\alpha=-1$,

$$
\begin{aligned}
R_{-1}(G) & =\sum_{e f \in E(G)} \frac{1}{\left(d_{e} \times d_{f}\right)} \\
R_{-1}(G) & =4(m+n+1) \frac{1}{(1 \times 6)}+(12 m n+8 m+8 n-4) \frac{1}{(2 \times 6)}+(6 m n-6 n) \frac{1}{(3 \times 6)} \\
& =\frac{4 m n+4 m+3 n+1}{3} .
\end{aligned}
$$

For $\alpha=-\frac{1}{2}$

$$
\begin{aligned}
R_{-\frac{1}{2}}(G) & =\sum_{e f \in E(G)} \frac{1}{\sqrt{\left(d_{e} \times d_{f}\right)}} \\
R_{-\frac{1}{2}}(G) & =4(m+n+1) \frac{1}{\sqrt{(1 \times 6)}}+(12 m n+8 m+8 n-4) \frac{1}{\sqrt{(2 \times 6)}}+(6 m n-6 n) \frac{1}{\sqrt{(3 \times 6)}} \\
& =(\sqrt{2}+2 \sqrt{3}) m n+2(m+n)\left(\frac{2 \sqrt{3}+\sqrt{6}}{3}\right)-\sqrt{2} n-\frac{2 \sqrt{3}}{3}+\frac{2 \sqrt{6}}{3} .
\end{aligned}
$$


For $\alpha=\frac{1}{2}$

$$
\begin{aligned}
R_{\frac{1}{2}}(G) & =\sum_{e f \in E(G)} \sqrt{\left(d_{e} \times d_{f}\right)} \\
R_{\frac{1}{2}}(G) & =4(m+n+1) \sqrt{(1 \times 6)}+(12 m n+8 m+8 n-4) \sqrt{(2 \times 6)}+(6 m n-6 n) \sqrt{(3 \times 6)} \\
& =6(3 \sqrt{2}+4 \sqrt{3}) m n+4(m+n)(4 \sqrt{3}+\sqrt{6})-18 \sqrt{2} n-8 \sqrt{3}+4 \sqrt{6} .
\end{aligned}
$$

\section{- The first, second, and hyper Zagreb indices}

Let $G \cong \operatorname{BiI}_{3}(m \times n)$ be a molecular graph of bismuth tri-iodide sheet. Now, using Table 3, the first, second, and hyper Zagreb indices are computed as

$$
\begin{aligned}
M_{1}(G) & =\sum_{e f \in E(G)}\left(d_{e}+d_{f}\right) \\
M_{1}(G) & =4(m+n+1)(1+6)+(12 m n+8 m+8 n-4)(2+6)+(6 m n-6 n)(3+6) \\
& =150 m n+92 m+38 n-4 . \\
M_{2}(G) & =\sum_{e f \in E(G)}\left(d_{e} \times d_{f}\right) \\
M_{2}(G) & =4(m+n+1)(1 \times 6)+(12 m n+8 m+8 n-4)(2 \times 6)+(6 m n-6 n)(3 \times 6) \\
& =252 m n+120 m+12 n-24 . \\
H M(G) & =\sum_{e f \in E(G)}\left(d_{e}+d_{f}\right)^{2} \\
H M(G) & =4(m+n+1)(1+6)^{2}+(12 m n+8 m+8 n-4)(2+6)^{2}+(6 m n-6 n)(3+6)^{2} \\
& =1254 m n+708 m+222 n-60 .
\end{aligned}
$$

Table 4 demonstrates the edge segment in view of the degree sum of the end vertices of each edge. We derived the exact result for the fourth atom-bond connectivity index and the fifth geometric arithmetic index using Table 4.

Table 4. Edge partition of $\mathrm{BiI}_{3}(m \times n)$ based on the degree sum of the end vertices of each edge.

\begin{tabular}{cc}
\hline$\left(S_{\boldsymbol{e}}, \boldsymbol{S}_{\boldsymbol{f}}\right)$ & Number of Edges \\
\hline$(6,10)$ & $4 n+8$ \\
$(6,12)$ & $4 m-4$ \\
$(10,12)$ & $8 n+16$ \\
$(12,12)$ & $16 m+12 n-28$ \\
$(12,14)$ & $12 m n-8 m-12 n+8$ \\
$(12,18)$ & $4 m-4$ \\
$(14,18)$ & $6 m n-4 m-6 n+4$ \\
\hline
\end{tabular}

- The fourth atom-bond connectivity index $A B C_{4}\left(B i I_{3}(m \times n)\right)$.

Let $G$ be the graph of silicon carbide of type $\mathrm{Bi}_{3}(m \times n)$. Now, using Table 4 , the fourth atom-bond connectivity index is computed as

$$
A B C_{4}(G)=\sum_{e f \in E(G)} \sqrt{\frac{S_{e}+S_{f}-2}{S_{e} \times S_{f}}} .
$$




$$
\begin{aligned}
A B C_{4}(G) & =4(n+2) \sqrt{\frac{6+10-2}{6 \times 10}}+4(m-1) \sqrt{\frac{6+12-2}{6 \times 12}}+8(n+2) \sqrt{\frac{10+12-2}{10 \times 12}} \\
& +(16 m+12 n-28) \sqrt{\frac{12+12-2}{12 \times 12}}+(12 m n-8 m-12 n+8) \sqrt{\frac{12+14-2}{12 \times 14}} \\
& +4(m-1) \sqrt{\frac{12+18-2}{12 \times 18}}+(6 m n-4 m-6 n+4) \sqrt{\frac{14+18-2}{14 \times 18}} \\
& =\left(\frac{12 \sqrt{7}+\sqrt{210}}{7}\right) m n+\left(\frac{4 \sqrt{2}}{3}-\frac{8 \sqrt{7}}{7}+\frac{4 \sqrt{22}}{3}+\frac{2 \sqrt{42}}{9}-\frac{2 \sqrt{210}}{21}\right) m \\
& +\left(\frac{4 \sqrt{6}}{3}-\frac{12 \sqrt{7}}{7}+\sqrt{22}-\frac{\sqrt{210}}{105}\right) n-\frac{4 \sqrt{2}}{3}+\frac{8 \sqrt{6}}{3}+\frac{8 \sqrt{7}}{7}-\frac{7 \sqrt{22}}{3}-\frac{2 \sqrt{42}}{9}+\frac{38 \sqrt{210}}{105} .
\end{aligned}
$$

- The fifth geometric arithmetic index $G A_{5}\left(B I_{3}(m \times n)\right)$.

Let $G$ be a molecular graph of bismuth tri-iodide sheet $\mathrm{BiI}_{3}(m \times n)$. Then, using Table 4 , the fifth geometric arithmetic index is computed as

$$
\begin{aligned}
G A_{5}(G) & =\sum_{e f \in E(G)} \frac{2 \sqrt{S_{e} \times S_{f}}}{S_{e}+S_{f}} \\
G A_{5}(G) & =4(n+2) \frac{2 \sqrt{6 \times 10}}{6+10}+4(m-1) \frac{2 \sqrt{6 \times 12}}{6+12}+8(n+2) \frac{2 \sqrt{10 \times 12}}{10+12} \\
& +(16 m+12 n-28) \frac{2 \sqrt{12 \times 12}}{12+12}+(12 m n-8 m-12 n+8) \frac{2 \sqrt{12 \times 14}}{12+14} \\
& +4(m-1) \frac{2 \sqrt{12 \times 18}}{12+18}+(6 m n-4 m-6 n+4) \frac{2 \sqrt{14 \times 18}}{14+18} \\
& =\left(\frac{112 \sqrt{7}+96 \sqrt{42}}{52}\right) m n+\left(\frac{8 \sqrt{2}}{3}+\frac{8 \sqrt{6}}{5}-\frac{3 \sqrt{7}}{2}-\frac{16 \sqrt{42}}{13}+16\right) m \\
& -\left(\frac{9 \sqrt{7}}{4}-\sqrt{15}-\frac{16 \sqrt{30}}{11}+\frac{24 \sqrt{42}}{13}-12\right) n \\
& -\frac{8 \sqrt{2}}{3}-\frac{8 \sqrt{6}}{5}+\frac{3 \sqrt{7}}{2}+2 \sqrt{15}+\frac{32 \sqrt{30}}{11}+\frac{16 \sqrt{42}}{13}-28 .
\end{aligned}
$$

\section{Comparisons and Discussion}

- $\quad$ For the comparison of these indices numerically for $m-B i I_{3}$, we computed all indices for different values of $m$. Now, from Table 5, we can easily see that all indices are in increasing order as the values of $m$ are increasing. The graphical representations of the topological indices for $m-\mathrm{BiI}_{3}$ are depicted in Figures 4-7 for certain values of $m$.

Table 5. All indices for $m-\mathrm{BiI}_{3}$.

\begin{tabular}{cccccccccccc}
\hline $\boldsymbol{m}$ & $\boldsymbol{A B C}$ & $\boldsymbol{R}_{\mathbf{1}}$ & $\boldsymbol{R}_{-\mathbf{1}}$ & $\boldsymbol{R}_{\frac{1}{2}}$ & $\boldsymbol{R}_{-\frac{1}{2}}$ & $\boldsymbol{G A}$ & $\boldsymbol{M}_{\mathbf{1}}$ & $\boldsymbol{M}_{\mathbf{2}}$ & $\boldsymbol{H} \boldsymbol{M}$ & $\boldsymbol{A B C _ { \mathbf { 4 } }}$ & $\boldsymbol{G A}_{\mathbf{5}}$ \\
\hline 1 & 26.5 & 288 & 4 & 49.5 & 8.5 & 45.5 & 276 & 360 & 2158 & 86.7 & 181.4 \\
2 & 34.8 & 552 & 6.2 & 102.8 & 15.9 & 58.9 & 464 & 624 & 3632 & 174.6 & 358.5 \\
3 & 52.3 & 816 & 8.3 & 145.2 & 22.8 & 98.7 & 652 & 888 & 5106 & 536.2 & 536.4 \\
4 & 85.4 .3 & 1080 & 11.4 & 192.5 & 30.5 & 142.5 & 840 & 1152 & 6580 & 704.2 & 704.3 \\
\hline
\end{tabular}




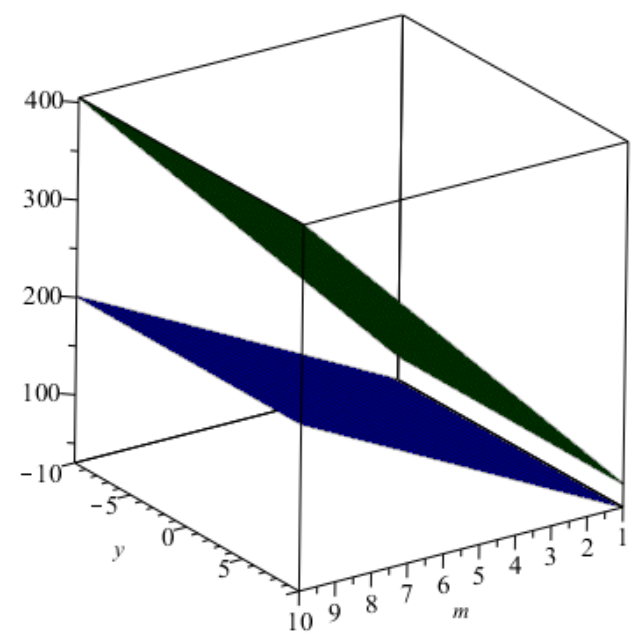

Figure 4. The graphical representation of $A B C\left(m-B i I_{3}\right)$ index in blue and that of the $G A\left(m-B i I_{3}\right)$ index in green.

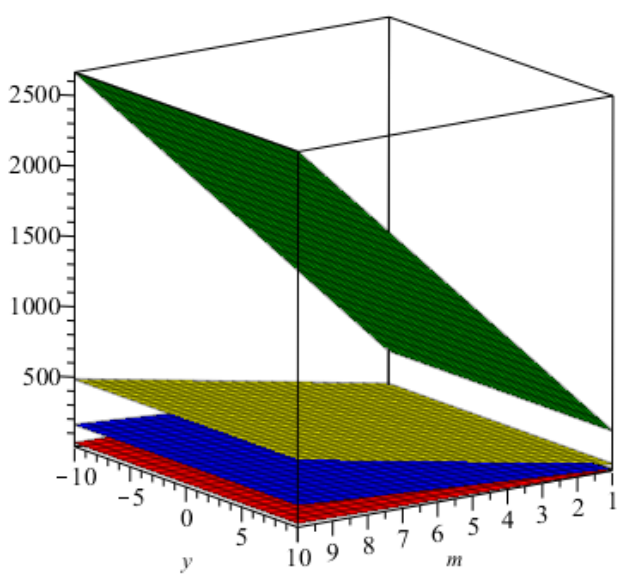

Figure 5. The graphical representation of the Randić index for $\alpha=1$ in green, that for $\alpha=-1$ in red, that for $\alpha=\frac{1}{2}$ in yellow, and that for $\alpha=-\frac{1}{2}$ in blue.

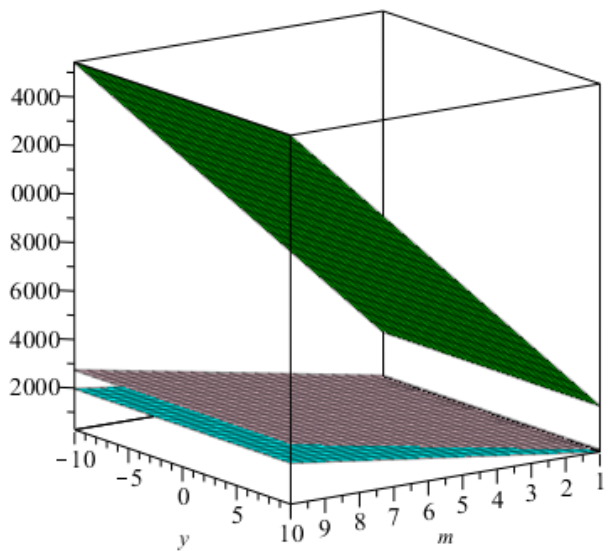

Figure 6. The graphical representation of the first, second, and hyper Zagreb indices in cyan, pink, and green, respectively. 


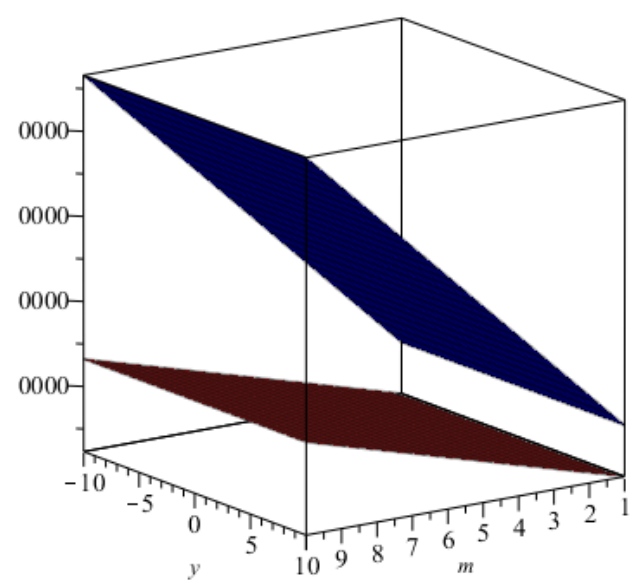

Figure 7. The graphical representation of $A B C_{4}\left(m-B i I_{3}\right)$ index in blue and that of the $G A_{5}\left(m-B i I_{3}\right)$ index in brown.

- We computed all indices numerically for $\operatorname{BiI}_{3}(m \times n)$ for different values of $m, n$. We can easily see, from Table 6 , that all indices are in increasing order as the values of $m, n$ are increasing. The graphical representations of the topological indices for $\operatorname{BiI}_{3}(m \times n)$ are depicted in Figures 8-11 for certain values of $m, n$.

Table 6. All indices for $\mathrm{BiI}_{3}(m \times n)$.

\begin{tabular}{cccccccccccc}
\hline$[\boldsymbol{m}, \boldsymbol{n}]$ & $\boldsymbol{A B C}$ & $\boldsymbol{R}_{\mathbf{1}}$ & $\boldsymbol{R}_{-\mathbf{1}}$ & $\boldsymbol{R}_{\frac{1}{2}}$ & $\boldsymbol{R}_{-\frac{1}{2}}$ & $\boldsymbol{G A}$ & $\boldsymbol{M}_{\mathbf{1}}$ & $\boldsymbol{M}_{\mathbf{2}}$ & $\boldsymbol{H} \boldsymbol{M}$ & $\boldsymbol{A B C _ { \mathbf { 4 } }}$ & $\boldsymbol{G} \boldsymbol{A}_{\mathbf{5}}$ \\
\hline$[1,1]$ & 14.2 & 360 & 3.4 & 7.6 & 81.2 & 13.2 & 276 & 360 & 2124 & 11.3 & 9.8 \\
{$[2,2]$} & 43.3 & 744 & 8.6 & 23.2 & 234.9 & 43.3 & 585 & 1248 & 6816 & 50.8 & 43.6 \\
{$[3,3]$} & 96.5 & 1128 & 19.2 & 45.8 & 565.3 & 96.5 & 1736 & 2640 & 14016 & 100.3 & 98.5 \\
{$[4,4]$} & 164.3 & 1412 & 31.4 & 64.3 & 965.4 & 164.8 & 2918 & 4536 & 23724 & 160.6 & 157.3 \\
\hline
\end{tabular}

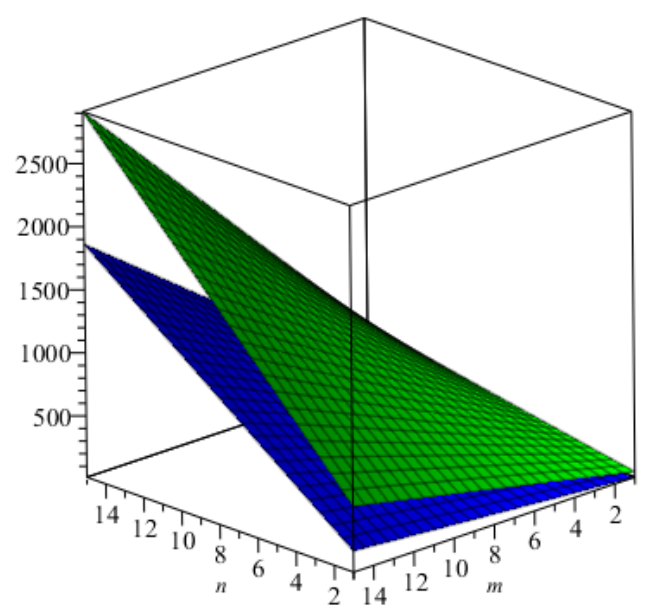

Figure 8. The graphical representation of the $A B C\left(B i I_{3}(m \times n)\right)$ index in green, and that of the $G A\left(\operatorname{BiI}_{3}(m \times n)\right)$ index in blue. 


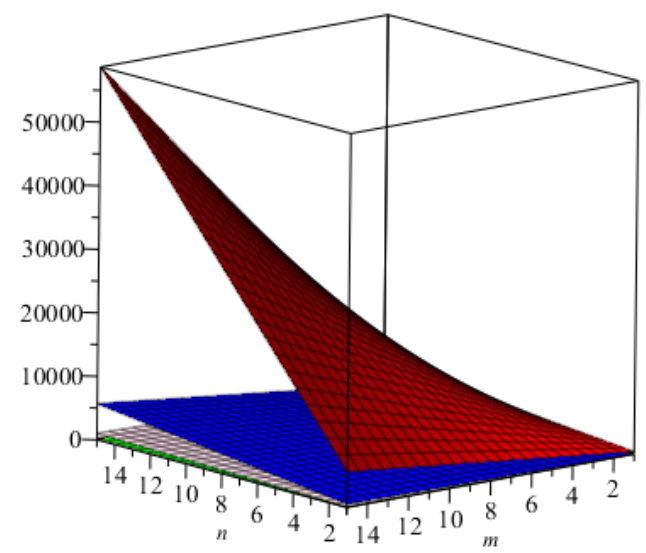

Figure 9. The graphical representation of the Randić index for $\alpha=1$ in red, that for $\alpha=-1$ in green, that for $\alpha=\frac{1}{2}$ in pink, and that for $\alpha=\frac{-1}{2}$ in blue.

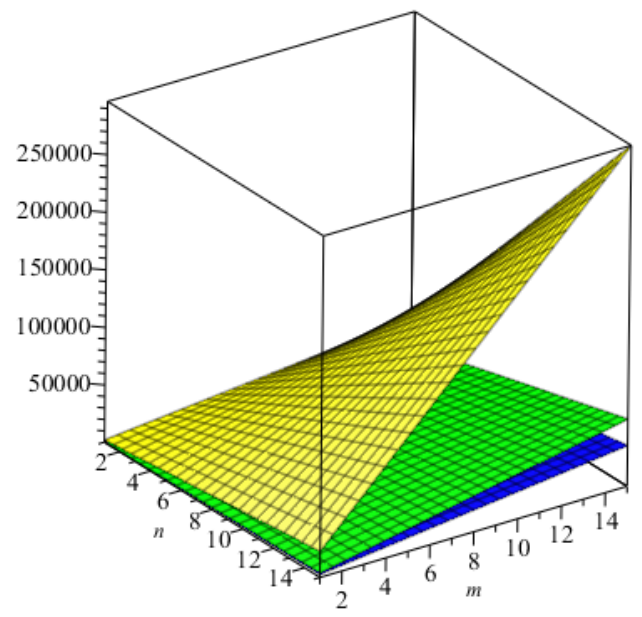

Figure 10. The graphical representation of the first, second, and hyper Zagreb indices in blue, green, and yellow, respectively.

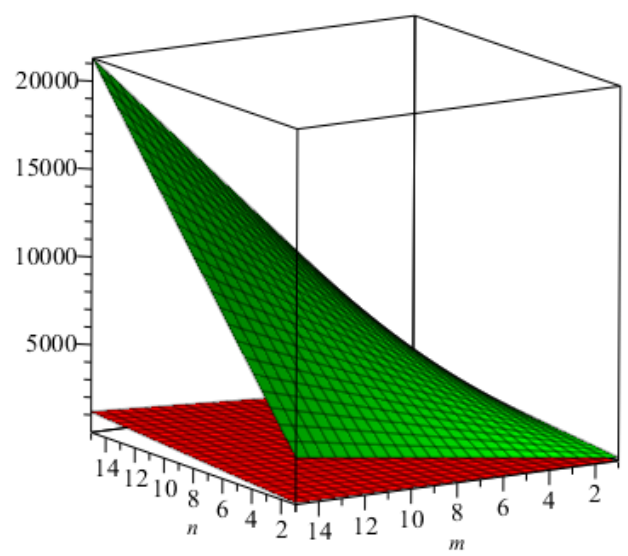

Figure 11. The graphical representation of $A B C_{4}\left(B i I_{3}(m \times n)\right)$ index in blue, and that of the $G A_{5}\left(\operatorname{BiI}_{3}(m \times n)\right)$ index in brown. 


\section{Conclusions}

We computed additive degree-based topological indices, the atom-bond network $A B C$ index, the general Randić index, the first, second, and hyper Zagreb indices, the arithmetic $G A$ index, the fourth atom-bond connectivity $A B C_{4}$ index, and the fifth geometric arithmetic $G A_{5}$ index of $m-\mathrm{BiI}_{3}$ and $\mathrm{BiI}_{3}(m \times n)$.

The Randic index is a topological descriptor that correlates with many chemical characteristics of molecules. It was found that the boiling point of $m-\mathrm{BiI}_{3}$ and $\mathrm{BiI}_{3}(m \times n)$ varies in increasing order for $\alpha \in\{1,-1,1 / 2,-1 / 2\}$.

The atom-bond connectivity $(A B C)$ index provides a very good correlation for computing the strain energy of molecules. One can easily see that the strain energy of $m-\mathrm{BiI}_{3}$ and $\mathrm{BiI}_{3}(m \times n)$ is high as the values of $m, n$ increase.

The GA index has as much predictive power as that of the Randic index, so the $G A$ index is more useful than the Randic index for $\alpha \in\{-1,-1 / 2\}$, as compared to the Randic index for $\alpha \in\{1,1 / 2\}$, in the case of $m-\mathrm{BiI}_{3}$ and $\mathrm{BiI}_{3}(m \times n)$.

The first and second Zagreb indices were found to occur for the computation of the total $\pi$-electron energy of molecules; thus, in the case of $m-\mathrm{BiI}_{3}$ and $\mathrm{BiI}_{3}(m \times n)$, their values provide the total $\pi$-electron energy in increasing order for higher values of $m, n$.

However, computation of distance-based and counting-related topological indices for these symmetrical chemical structures are open challenges and have yet to be investigated.

Author Contributions: M.I. contribute for conceptualization, designing the experiments, funding, supervision and analyzed the data. M.K.S. contribute for methodology, software, validation, and formal analysing. M.A.A. and S.A. contribute for performed experiments, resources, some computations and wrote the initial draft of the paper which were investigated and approved by M.I., M.K.S. and A.Q.B. and wrote the final draft. All authors read and approved the final version of the paper.

Funding: This research is supported by the Start-Up Research Grant 2016 of United Arab Emirates University (UAEU), Al Ain, United Arab Emirates via Grant No. G00002233 and UPAR Grant of UAEU via Grant No. G00002590.

Acknowledgments: The authors are grateful to the anonymous referees for their valuable comments and suggestions that improved this paper.

Conflicts of Interest: The authors declare no conflict of interest.

\section{References}

1. Randic, M. On characterization of molecular branching. J. Am. Chem. Soc. 1975, 97, 6609-6615. [CrossRef]

2. Bollobas, B.; Erdos, P. Graphs of extremal weights. Ars Comb. 1998, 50, 225-233. [CrossRef]

3. Amic, D.; Beslo, D.; Lucic, B.; Nikolic, S.; Trinajstić, N. The vertex-connectivity index revisited. J. Chem. Inf. Comput. Sci. 1998, 38, 819-822. [CrossRef]

4. Li, X.; Gutman, I. Mathematical Aspects of Randić Type Molecular Structure Descriptors; Mathematical Chemistry Monographs, No. 1; University of Kragujevac and Faculty of Science: Kragujevac, Serbia, 2006; ISBN 86-81829-67-X.

5. Estrada, E.; Torres, L.; Rodríguez, L.; Gutman, I. An atom-bond connectivity index: Modelling the enthalpy of formation of alkanes. Indian J. Chem. 1998, 37A, 849-855.

6. Gutman, I.; Trinajstc, N. Graph theory and molecular orbitals, Total $\pi$-electron energy of alternant hydrocarbons. Chem. Phys. Lett. 1972, 17, 535-538. [CrossRef]

7. Gao, W.; Siddiqui, M.K.; Naeem, M.; Rehman, N.A. Topological Characterization of Carbon Graphite and Crystal Cubic Carbon Structures. Molecules 2017, 22, 1496. [CrossRef] [PubMed]

8. Siddiqui, M.K.; Imran, M.; Ahmad, A. On Zagreb indices, Zagreb polynomials of some nanostar dendrimers. Appl. Math. Comput. 2016, 280, 132-139. [CrossRef]

9. Siddiqui, M.K.; Naeem, M.; Rahman, N.A.; Imran, M. Computing topological indicesof certain networks. J. Optoelectron. Adv. Mater. 2016, 18, 884-892.

10. Shirdel, G.H.; RezaPour, H.; Sayadi, A.M. The Hyper-Zagreb Index of Graph Operations. Iran. J. Math. Chem. 2013, 4, 213-220. 
11. Ghorbani, M.; Hosseinzadeh, M.A. Computing $A B C_{4}$ index of nanostar dendrimers. Optoelectron. Adv. Mater-Rapid Commun. 2010, 4, 1419-1422.

12. Vukicevic, D.; Furtula, B. Topological index based on the ratios of geometrical and arithmetical means of end-vertex degrees of edges. J. Math. Chem. 2009, 46, 1369-1376. [CrossRef]

13. Graovac, A.; Ghorbani, M.; Hosseinzadeh, M.A. Computing fifth geometric arithmetic index for nanostar dendrimers. J. Math. Nanosci. 2011, 1, 33-42.

14. Shao, Z.; Wu, P.; Zhang, X.; Dimitrov, D.; Liu, J. On the maximum $A B C$ index of graphs with prescribed size and without pendent vertices. IEEE Access. 2018, doi 10.1109/access.2018.2831910. [CrossRef]

15. Shao, Z.; Wu, P.; Gao, Y.; Gutman, I.; Zhang, X. On the maximum $A B C$ index of graphs without pendent vertices. Appl. Math. Comput. 2017, 315, 298-312. [CrossRef]

16. Das, K.C.; Gutman, I.; Furtula, B. Survey on geometric-arithmetic indices of graphs. MATCH Commun. Math. Comput. Chem. 2011, 65, 595-644.

17. Gutman, I.; Ruscic, B.; Trinajstić, N.; Wilcox, C.F. Graph theory and molecular orbitals, XII. Acyclic polyenes. J. Chem. Phys. 1975, 62, 3399-3405. [CrossRef]

18. Schlter, M.; Cohen, M.L.; Kohn, C.E.; Fong, C.Y. Electronic Structure of BiI $I_{3}$. Phys. Status Solidi (B) 1976, 78. [CrossRef]

19. Gao,W.; Farahani, M.R. Degree-based indices computation for special chemical molecular structures using edge dividing method. Appl. Math. Nonlinear Sci. 2015, 1, 94-117. [CrossRef]

20. Gao, W.; Siddiqui, M.K. Molecular Descriptors of Nanotube, Oxide, Silicate, and Triangulene networks. J. Chem. 2017, 2017, 6540754. [CrossRef]

21. Gao, W.; Siddiqui, M.K.; Imran, M.; Jamil, M.K.; Farahani, M.R. Forgotten topological index of chemical structure in drugs. Saudi Pharm. J. 2016, 24, 258-264. [CrossRef] [PubMed]

22. Baig, A.Q.; Imran, M.; Khalid, W.; Naeem, M. Molecular description of carbon graphite and crystal cubic carbon structures. Can. J. Chem. 2017, 95, 674-686. [CrossRef]

23. Baig, A.Q.; Imran, M.; Ali, H. On topological indices of poly oxide, poly silicate, DOX, and DSL networks. Can. J. Chem. 2015, 93, 730-739. [CrossRef]

24. Hayat, S.; Imran, M. Computation of certain topological indices of nanotubes covered by $C_{5}$ and $C_{7}$. J. Comput. Theor. Nanosci. 2015, 12, 533-541. [CrossRef]

25. Iodide. B. McGraw-Hill Dictionary of Scientific and Technical Terms; McGraw-Hill: New York, NY, USA, 2003.

26. Mackay, R.A.; Henderson, W. Introduction to Modern Inorganic Chemistry; CRC Press: Boca Raton, FL, USA, 2002; pp. 122-126, ISBN 0-7487-6420-8.

27. Smart, L.; Elaine, A.M. Solid State Chemistry: An Introduction; CRC Press: Boca Raton, FL, USA, 2005; p. 40, ISBN 0-7487-7516-1.

28. Watanabe, K.; Karasawa, T.; Komatsu, T.; Kaifu, Y. Optical Properties of Extrinsic Two-Dimensional Excitons in $\mathrm{BiI}_{3}$ Single Crystals. J. Phys. Soc. Jpn. 1986, 55. [CrossRef]

29. Wyckoff, R.W.G. Crystal Structures, 2nd ed.; John Wiley \& Sons, Inc.: New York, NY, USA; London, UK; Sydney, Australia, 1964.

30. Yorikawa, H.; Muramatsu, S. Theoretical Study of Crystal and Electronic Structures of BiI $I_{3}$ J. Phys. Condens. Matter 2008, 20, 325-335. [CrossRef]

31. Nason, D.; Keller, L. The Growth and Crystallography of Bismuth Tri-Iodide Crystals Grown by Vapor Transport. J. Cryst. Growth 1995, 221. [CrossRef]

32. Erdmann, H.; Dunlap, F.L. Handbook of Basic Tables for Chemical Analysis; John Wiley \& Sons: New York, NY, USA, 1900.

(C) 2018 by the authors. Licensee MDPI, Basel, Switzerland. This article is an open access article distributed under the terms and conditions of the Creative Commons Attribution (CC BY) license (http:/ / creativecommons.org/licenses/by/4.0/). 